\title{
Design and Implementation of a Biometric Students' Time and Attendance Logging System
}

\author{
D. U. Onyishi*, C. K. Igbinoba \\ ${ }^{a}$ Department of Electrical and Electronics Engineering, Federal University of Petroleum Resources Effurun, \\ Delta State, NIGERIA.
}

\begin{abstract}
Every tertiary academic institution has the obligation of recording and taking the attendance of its students during lecture periods. Over the years the process and accuracy of this attendance has been marred by many challenges which range from the cumbersome nature of paper sheets used in recording, to the manipulation of attendance by unscrupulous students and so on. This paper is aimed at designing and implementing a biometric students' time and attendance logging system that helps in mitigating these challenges. This biometric student time and attendance logging system uses the fingerprint biometric characteristics to accurately identify the student. The system also generates a report showing the number of times a particular student was present or absent in a semester. This feat was achieved through the use of an atmega328P microcontroller, a fingerprint RS305 module, a liquid crystal display (LCD), a Red Green Blue (RGB) light emitting diode (LED) and a Personal Computer (PC) amongst others. The software used in this work was developed using visual studio C\# and MySQL2008, and the final work was simulated using proteus before it was constructed and tested. The results of the test show a significant improvement over the manual attendance system.
\end{abstract}

Keywords: Atmega328P Microcontroller, attendance, biometric, fingerprint, RS305 module

\section{INTRODUCTION}

The act of taking attendance is a very vital process in academic institutions, organizations, companies etc. In early days and even till now attendance is taken manually in academic institutions by calling out names or by passing attendance sheets for the students to mark [1]. But this process can be easily exploited by fraudulent students who answer or sign in present for their friends who are not present in attendance. Therefore an improved attendance system is needed to prevent such fraudulent activities. The use of a Biometric time and attendance logging system would greatly help in increasing the reliability and accuracy of attendance taking. A Biometric is a biological and behavioral characteristics of an individual from which distinguishing, repeatable biometric features can be extracted for the purpose of biometric recognition [2]. Some of the biological or physiological biometric characteristics include fingerprint, face, iris, retina, hand geometry, palm print, ear, facial thermogram, DNA. While the behavioral characteristics include dynamic signature, keystroke dynamics, voice and gait [3]. Biometric recognition systems operate in

${ }^{*}$ Corresponding author (Tel: +234(0) 803432 1196)

Email addresses: onyishi.donatus@fupre.edu.ng (D. U. Onyishi), igbinoba.charles@fupre.edu.ng (C. K. Igbinoba) two modes namely verification and identification. In verification mode, the system checks whether a user is the person who owns the biometric at the point of access to a resource. The system carries out a $1: 1$ or one to one matching and this mode answers the question "is the user who he claims to be?" [4]. In the identification mode a 1: $\mathrm{N}$ or one to many comparison is made [4], here the system recognizes an individual by searching the templates of all users in the database for a match [5].

The fingerprint biometric is the most widely deployed trait in biometric recognition due to its acceptability to users as well as easy and cheap integration in existing and new systems [4]. A fingerprint is the array of ridges and valleys on the surface of a fingertip, the formation which is determined during the first seven months of fetal development [5]. These ridges are also called frictional ridges and they help the hand grasp objects by increasing friction and improving tactile sensing of surface textures. One major important use of friction ridges is in person identification due to the fact that the pattern of friction ridges on each finger is unique and immutable, enabling its use as a mark of identity [6]. Over the years, various research and works have been carried out by various researchers and scientist on time and attendance marking system. Selv et al. [7] used a face biometric recognition attendance marking system to take the attendance of 


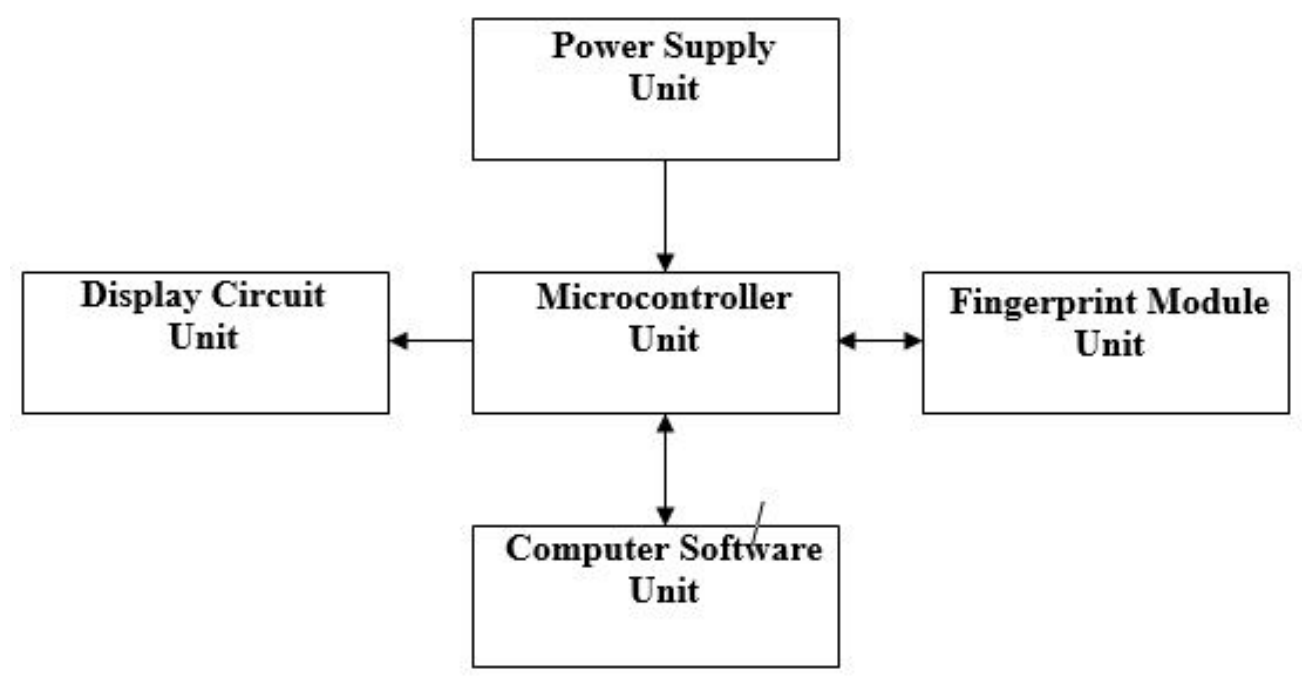

Figure 1: Block diagram of the biometric time and attendance logging system.

individuals. Freya et al. [8] carried out an android based attendance system, where by an android application "MAttS" was created to assist in attendance taking. Palavi and Namit [9] carried out a fingerprint based attendance system using Global System for Mobile Communication (GSM), in this case the system takes the attendance with the aid of a fingerprint processor, then at the end of the attendance taking exercise the system then sends the attendance of every student to their parent's mobile through GSM. Also Sumita et al. [10] carried out work on attendance management by using a Radio Frequency Identification Technology (RFID) which comprises of RFID tags, an Antenna, RFID Readers, RFID Middleware, a Backend Database amongst others, to take attendance of individuals.

\section{METHODOLOGY}

The methodology employed in this system uses a data and process partitioning technique to improve speed of processing. The data and process partitioning was implemented as follows;

- By using the powerful SyncoCHIP AS601 DSP processor to render the template image, perform calculations.

- By using ASIC PS1802 to store fingerprint templates, assign Address for the template and match fingerprint templates.

- By using Atmega 328P-PU to store the program firmware, control other Peripherals which are necessary for the system operation and serve as a UART interface between the AS601 and the computer.

- By using a Software Program whose function is to store students' data, match students data with their fingerprint template, provide a friendly UI (User Interface) for the system, Logging and Analysis of students Attendance.
The above technique improved the speed of the whole system because of the division of labour.

\section{DESIGN ANALYSIS}

The block diagram of the biometric students' time and attendance logging system is shown in Fig. 1.

The Biometric time and attendance logging system consist of 5 major units namely;

- Power Supply Unit

- Microcontroller Unit

- Display Unit

- Fingerprint Module Unit

- Computer Software Unit

\subsection{Power Supply Unit}

The whole circuit is powered from the Personal Computer (PC) through a Universal Asynchronous Receiver Transmitter (UART) to Universal Serial Bus (USB) cable, i.e. a UART to USB cable. The UART end of the cable consist of 4 wires, 2 of which serve as receiver and transmitter lines while the other 2 wires serve as power lines supplying the 5 Volts needed by the atmega328P microcontroller. The UART end is connected to the microcontroller while the USB end is connected to the PC.

\subsection{Microcontroller Unit}

This is the heart of the full circuit as it interfaces with all other units. The microcontroller used was the Atmega328P. The Atmega328P is a 28 pin microcontroller. It consists of a $32 \mathrm{~kb}$ flash memory for program storage, a $2 \mathrm{~kb}$ Random Access Memory (RAM), 6 channels of 10-bit analog to digital converter (ADC), $1 \mathrm{~kb}$ EEPROM Memory, two 8 bit and one 16 bit timer/counter and serial communication ports for communication with the 
COM port of a computer. The microcontroller controls the LCD on what message to display, it also controls the RGB LED on what colour to display and when to display it. The microcontroller also manages the interaction between the R305 fingerprint module and the PC. The microcontroller has two $22 \mathrm{pF}$ resonant capacitor and a $16 \mathrm{MHz}$ crystal oscillator connect to it.

\subsection{Display Unit}

This unit consists majorly of a Liquid Crystal Display (LCD) and an RGB Light Emitting Diode (LED). Both the LCD and the LED are connected to the Atmega328p microcontroller. The RGB LED is connected through a 220 ohms resistors to pin 17, 18 and 19 of the atmega328P. The LCD used is a HITACHI HD44780 1602 LCD. The pin 5 of the LCD is the Read/Write (R/W) pin. When the R/W terminal is set high, it means a Read Operation is to be initiated, also when the $\mathrm{R} / \mathrm{W}$ is set low it means a Write Operation is to be initiated. So therefore the R/W terminal is permanently grounded in this work because we only want to write into the LCD. The LCD is also configured to operate in a 4-bit mode. The RGB LED shows RED when a Non-match occurs, it shows GREEN when a match occurs and the RGB LED blinks BLUE when the system is processing a fingerprint. The LCD displays "Processing" when a fingerprint is being processed and it also displays the name of the student once a match is confirmed.

\subsection{Fingerprint Module}

The fingerprint module used was the RS305 fingerprint module. This module uses a very powerful SyncoCHIP AS601 DSP processor. AS601 is a member of the Synochip's Cordis 5+ family, directed for Fingerprint Identification and Digital Certification and can be used in a wide collection of embedded applications. AS601 works on typical frequency of $120 \mathrm{MHz}$. It provides a set of high-performance peripheral components including $128 \mathrm{~kb}$ SRAM, $64 \mathrm{~kb}$ ROM, $4 \mathrm{~kb}$ OTP memory, USB 2.0 (FS) Device, $2 \times$ USARTs, Limited Optical CMOS Sensor Controller (LOCSC), Asynchronous Parallel Controller (APC), RSA engine, Pseudo/True Random Number Generator(RNG), and up to 37 GPIOs.

The RS305 module is connected to the Receiver (Rx) and Transmitter (Tx) Pin of the Atmega328P microcontroller which are Pin 2 and Pin 3 respectively. The RS305 operates in 2 different modes namely fingerprint enrollment and fingerprint matching.

\subsubsection{Fingerprint enrollment}

This mode entails the initial acquisition and registration of the student's fingerprint template. The RS305 Fingerprint module initially scans the student's Fingerprint. The finger biometric template is then extracted from the scanned fingerprints and stored in a register in the RS305 module. The Atmega328P which is connected to the RS305 senses that a template has been stored and then sends a signal to the Personal Computer (PC) software to notify it that a template has been saved. This process is then repeated for each student until all the student's fingerprint template has be extracted and stored.

\subsubsection{Fingerprint matching}

In this mode, the student is required to place his/her initially registered finger on the RS305 fingerprint module. The RS305 module contains a powerful processor and matching algorithm that scans the student's current fingerprint and searches for a match among the already stored fingerprint template in the RS305 database. The result of this search (match or non-match) is then sent to the Atmega328P microcontroller.

\subsection{Computer Software}

A computer database software was created to store various information about the students. The computer is connected to the Atmega328P microcontroller through a UART to USB cable. The receiver (Rx) and transmitter (Tx) pin of the microcontroller (i.e pin 2 and pin 3) has already been used by the RS305, so therefore two I/O pins were converted to serve as Rx and Tx pins so that they can be connected to the UART side of the cable. During the fingerprint enrollment, once a student's template has been successfully stored in the RS305, the microcontroller then sends a signal to the PC software telling it that a fingerprint template has been stored in a particular register of the RS305. The PC Administrator with the aid of the software then assigns a name to that particular template stored in that particular register. In addition to the name, other personal data's such as gender and matriculation number are also saved with respect to that particular fingerprint template in that particular register as shown in Fig. 2.

During the fingerprint matching, the microcontroller sends signals to the PC's software to specify if a Match or Non-match of fingerprint has occurred. If the result is a match, the software marks the student present, also if the result is a Non-match the software marks the student absent. Also with the aid of the computer software, the students' data such as name, gender and matriculation number can be managed or edited and a report containing the number of times a student was present or absent can be generated.

The software requires a login password to prevent unauthorized persons from accessing it. So therefore only the Administrator has access to the software as shown in Fig. 3.

\section{FLOW CHART}

The working algorithm of the biometric and time attendance logging system is represented by the flow chart shown in Fig. 4.

\section{OPERATING PRINCIPLE SYSTEM}

The circuit diagram of the biometric and time attendance logging system is shown in Fig. 5. It 


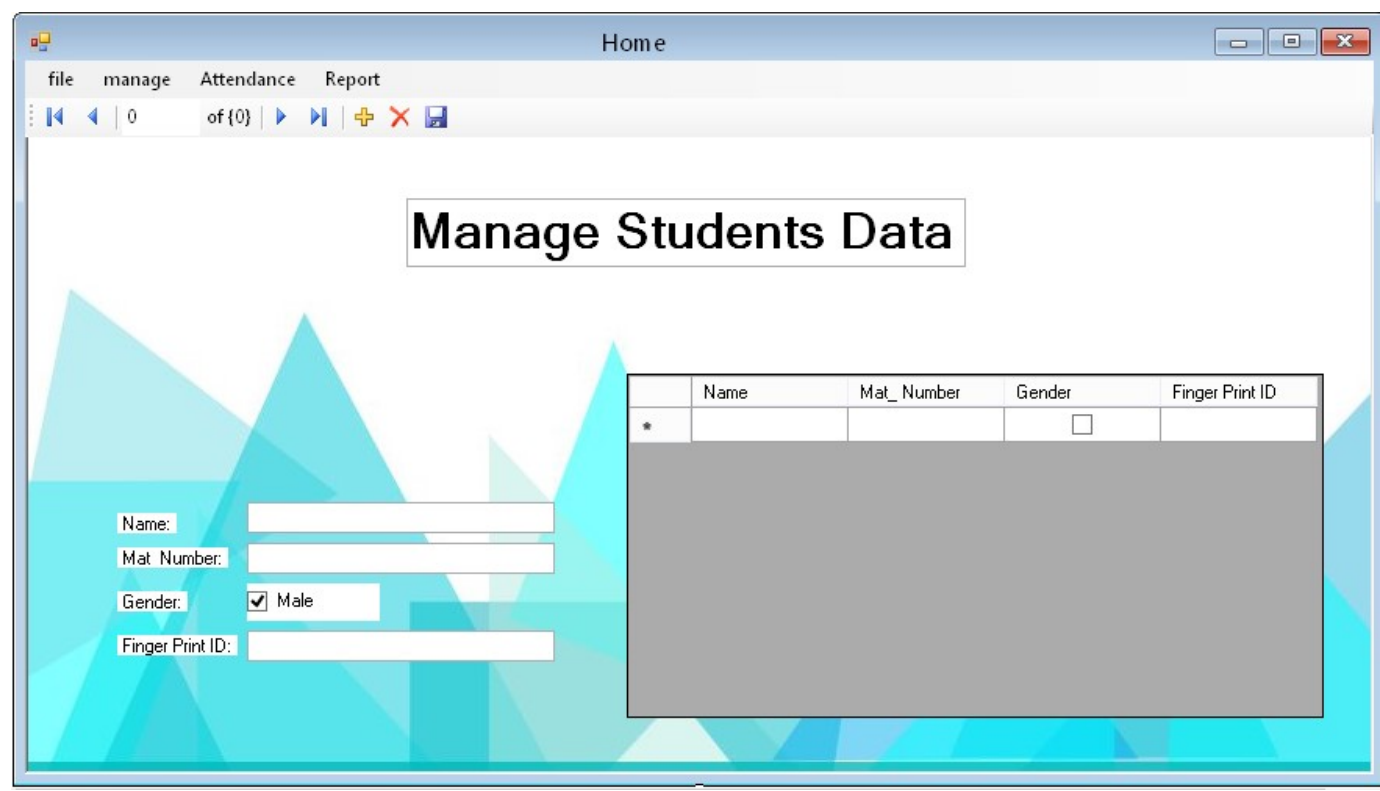

Figure 2: Pictorial view of software user interface for managing students data.

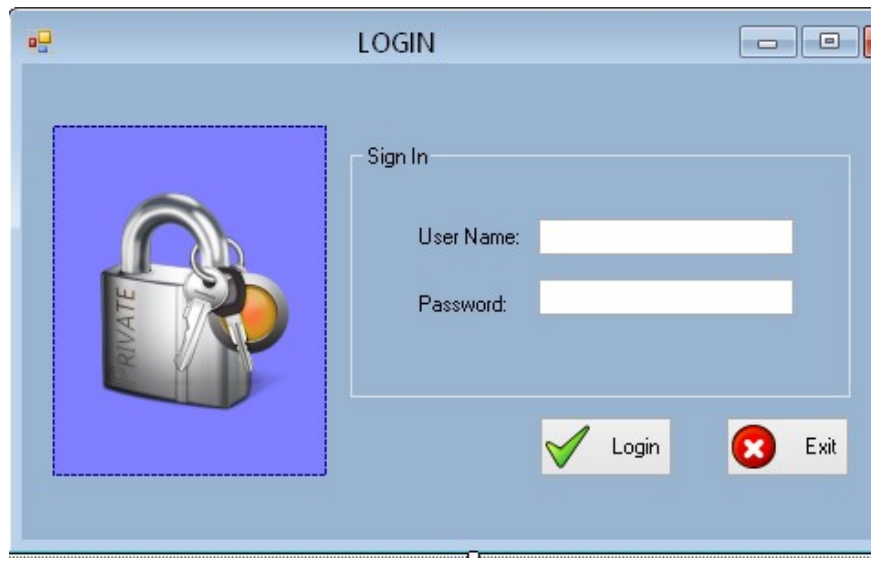

Figure 3: Image of the password login form.

basically operates in 2 phases namely, Registration and Identification.

During the registration phase each students fingerprint is scanned and the fingerprint template is extracted and stored in the RS305 module. In addition to the scanned fingerprint other details such as name, gender and matriculation number are collected and stored in the PC software database. It is also important to note that an option to manage and edit the already saved students' information is available with the aid of the computer software. For each time a fingerprint template is being extracted and saved, the LCD displays the word "processing" and the RGB LED blinks BLUE. After the successful registration of all the students, the next phase is the Identification phase.

In the Identification phase the student's fingerprint is required to be scanned by the RS305 fingerprint module. The RS305 module scans the current fingerprint and searches its database for a match, during this process the LCD displays the word "processing" and the RGB LED blinks BLUE. If a match occurs, the LCD then displays the name of the student and the PC software marks the students present whilst also showing other details of the student such as the students' name, gender and matriculation number. Also the RGB LED shows GREEN when a fingerprint match occurs. But if after the RS305 searches its database and a match could not be found i.e a Nonmatch, the RGB LED would show RED, meaning either the student wasn't registered or a false Non-match has occurred.

At the end of the semester or after a particular period of time as determined by the lecturer, a report showing the number of times a student has been present or absent can be generated with the aid of the computer software.

\section{TESTING, RESULT AND ANALYSIS}

After the successful design and implementation of the circuit, the system was tested using the biodata and fingerprints collected from twenty (20) students. In the test, there was no false recognition i.e. a person that was not initially registered was not falsely enrolled for attendance. There were some false rejections during the testing where the system failed to identify some preregistered users. The false rejects could be ascribed to improper placement of the finger on the scanner and fingers that have been slightly scarred due to injuries. The test of the 20 students was carried out 8 times in groups of 10 and a success rate of about $95 \%$ was obtained from the tests carried out. The results obtained from the test carried out are shown in Table 1 and Fig. 6 .

From the test, the total period to register a new user i.e. extract the information from the fingerprint and input the bio-data is about 1 minute 20 seconds. For the actual attendance collection process, the total time taken to extract the information from the fingerprint, identify the user and 


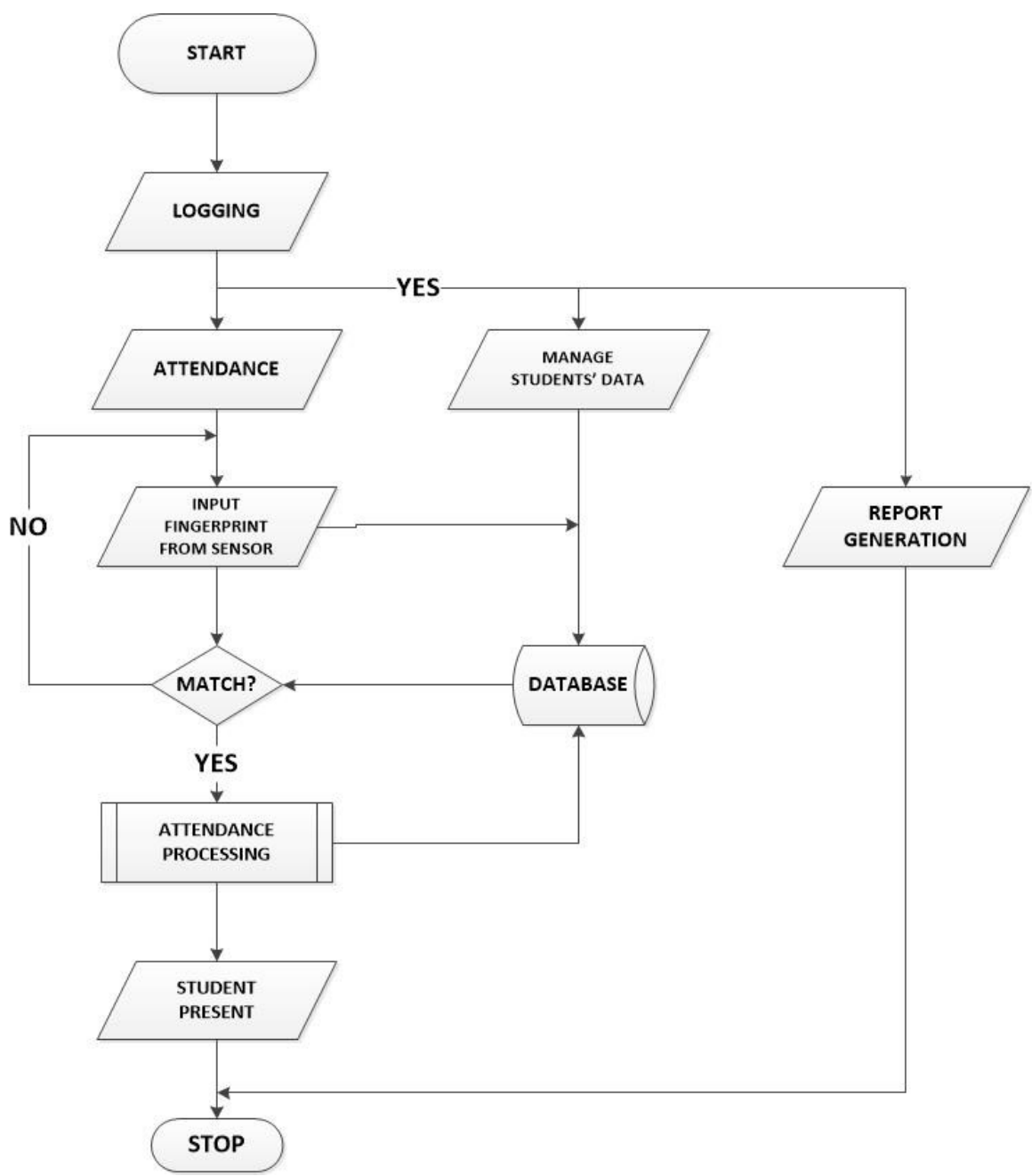

Figure 4: Software flow chart.

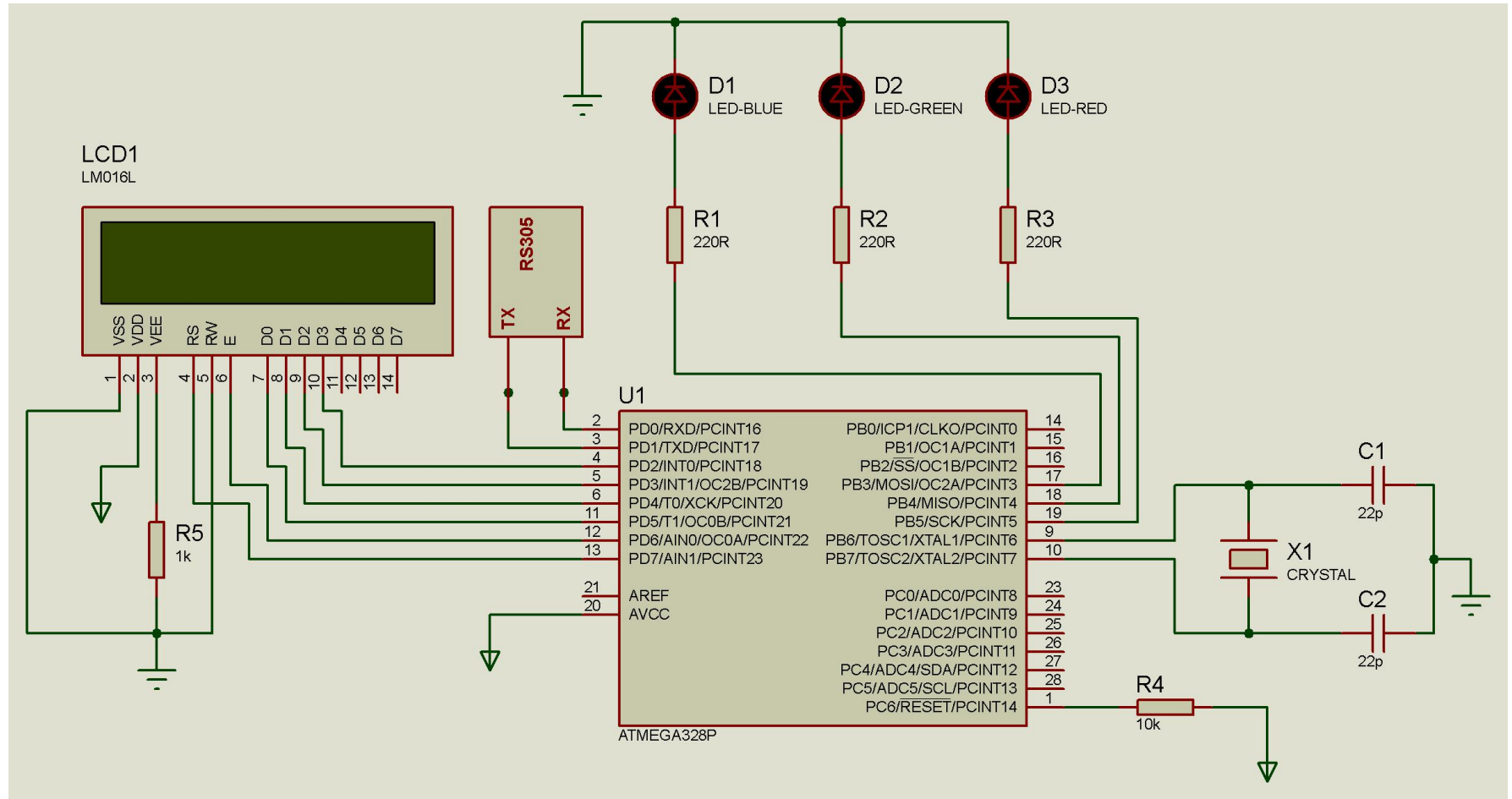

Figure 5: Complete Circuit Diagram of a Biometric Students' Time and Attendance Logging System. 
Table 1: Table showing percentage success/failure.

\begin{tabular}{llllllllc}
\hline Groups & 1 & 2 & 3 & 4 & 5 & 6 & 7 & 8 \\
Success (\%) & 100 & 90 & 100 & 100 & 80 & 100 & 90 & 100 \\
Failure (\%) & 0 & 10 & 0 & 0 & 20 & 0 & 10 & 0 \\
\hline
\end{tabular}

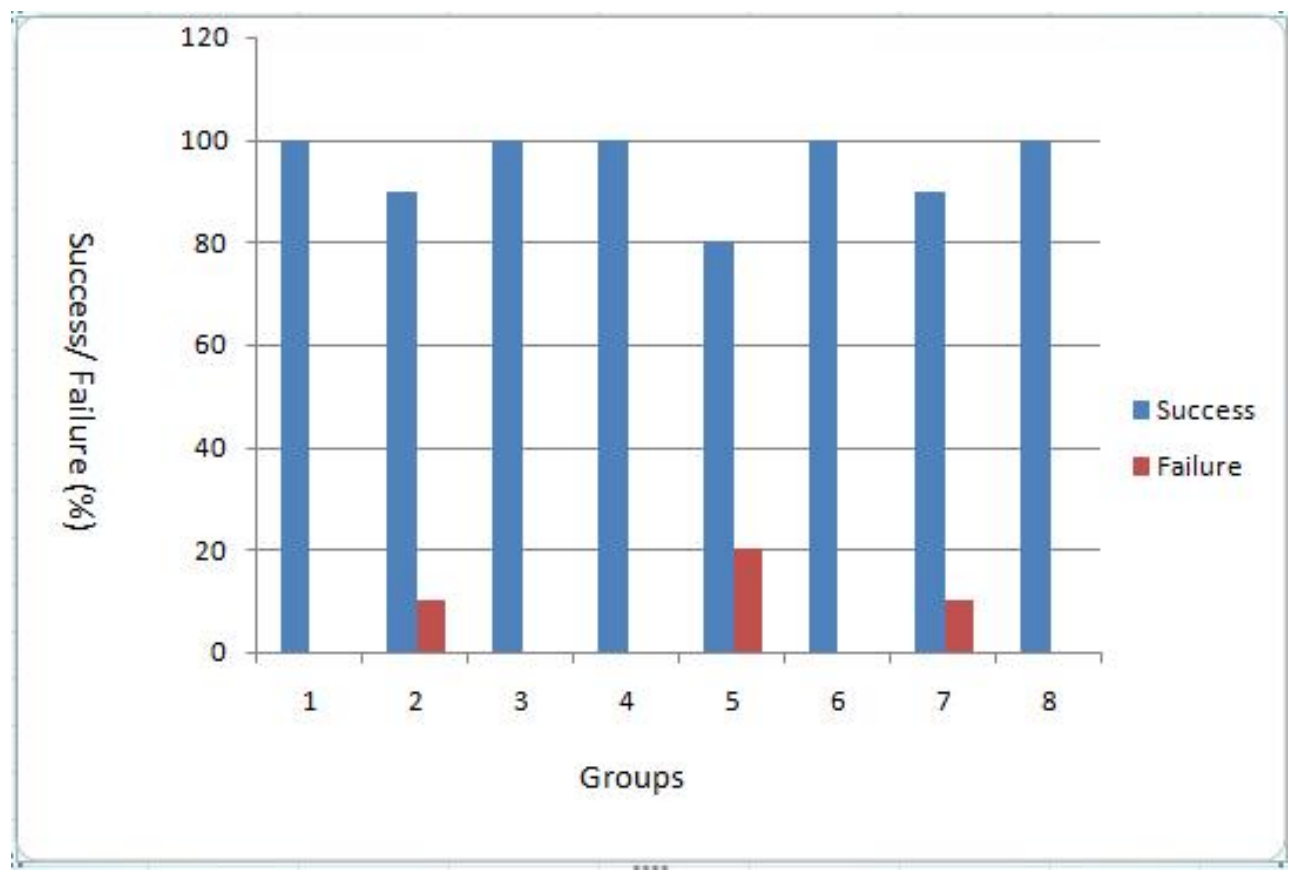

Figure 6: Graph showing percentage success/failure.

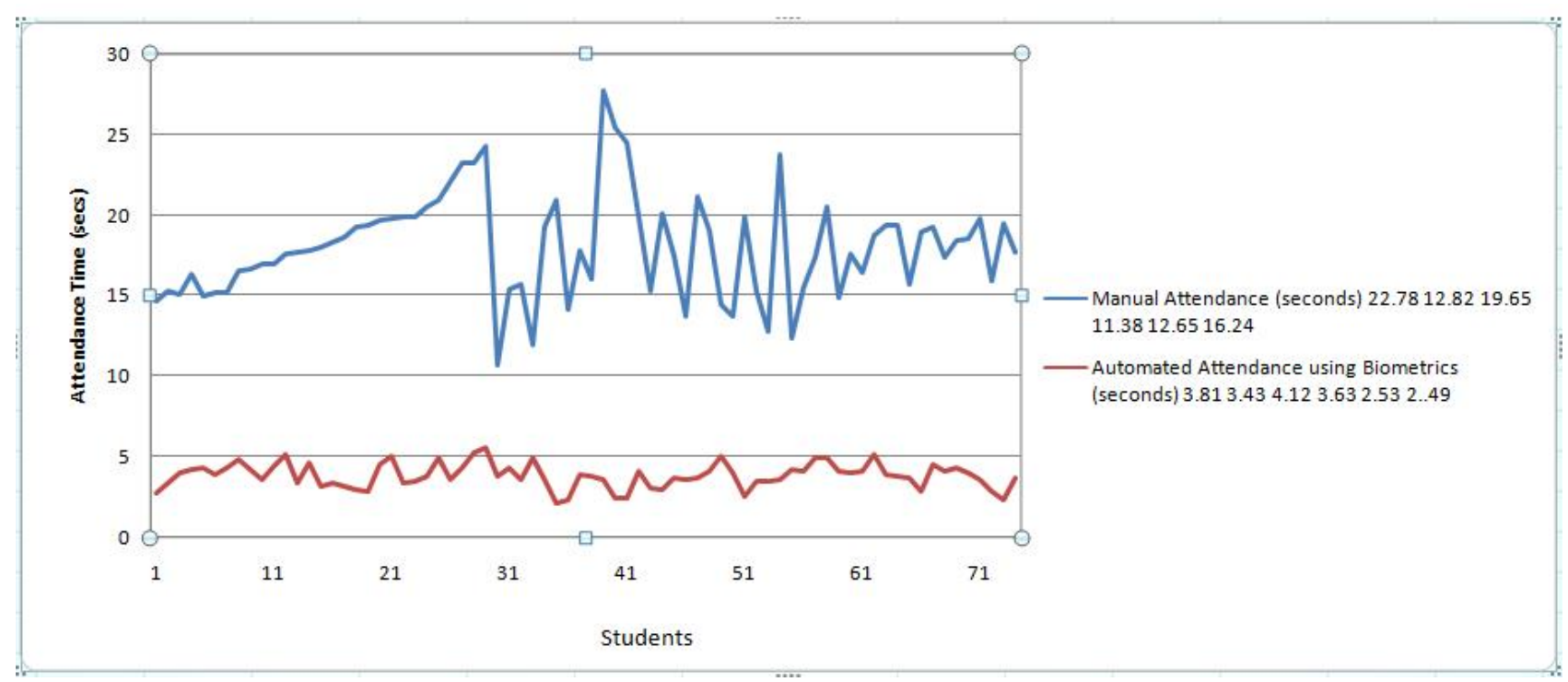

Figure 7: Graphical comparison between manual and automated attendance system. 
record the attendance for that particular course is less 5 seconds. Therefore, the system can be effectively used in classes with any type of population.

Table 2: Tabular comparison between manual and automated attendance system.

\begin{tabular}{llc}
\hline Student & Manual & Automated \\
\hline 1 & 22.78 & 3.81 \\
2 & 12.82 & 3.43 \\
3 & 19.65 & 4.12 \\
4 & 11.38 & 3.63 \\
5 & 12.65 & 2.53 \\
6 & 16.24 & 2.49 \\
7 & 14.66 & 2.72 \\
8 & 15.23 & 3.35 \\
9 & 15.03 & 4.01 \\
10 & 16.31 & 4.21 \\
11 & 14.97 & 4.31 \\
12 & 15.16 & 3.85 \\
13 & 15.18 & 4.32 \\
14 & 16.54 & 4.78 \\
15 & 16.59 & 4.23 \\
16 & 16.92 & 3.55 \\
17 & 16.95 & 4.34 \\
18 & 17.61 & 5.11 \\
19 & 17.72 & 3.36 \\
20 & 17.78 & 4.57 \\
\hline
\end{tabular}

\section{COMPARISON WITH MANUAL ATTEN- DANCE}

Table 2 shows a tabular comparison between the automated attendance management system using fingerprint authentication and the manual attendance system. From the table, it can be seen that the average execution time for twenty (20) students is approximately 19.19 seconds for the manual attendance as against 3.84 seconds for the automated attendance management system using fingerprint identification, Fig. 7 shows the graphical comparison between the manual and automated attendance system.

\section{CONCLUSION}

The system successfully simulated students' attendance and time logging. The prototype successfully captured fresh fingerprints to be stored in the database; scanned fingerprints placed on the device sensor and compared them against those stored in the database successfully. The average execution time for 20 students was 3.84 seconds and the system showed a $95 \%$ success rate.

\section{References}

[1] K. Karthik, S. Mary, B. Sumalatha, and A. Pereira, "Fingerprint based attendance system," International Journal of Advance research in Computer and Communication Engineering, vol. 4, no. 3, pp. 621-623, 2015.

[2] ISO, "Information technology - vocabulary-part 37: Biometrics," ISO/IEC 2382-37:2012(E), 2012, switzerland.

[3] M. David, M. Dario, K. Anil, and P. Salil, Handbook of Fingerprint Recognition, 2nd ed. Springer, 2009.

[4] O. Iloanusi, C. Osuagwu, and E. Osuagwu, Fundamentals of Biometrics, 1st ed. University of Nigeria Press, 2015.
[5] A. Jain, A. Ross, and S. Prabhakar, "An introduction to biometrics recognition," IEEE Transaction on Circuit and Systems for Video Technology, special issue on Image and Video based Biometic, vol. 14, no. 1, pp. 4-20, January 2004

[6] K. Anil, F. Jianjiang, and N. Karthik, "Fingerprint Matching, Washington," IEEE Computer Society, pp. 36-44, 2010.

[7] K. Selvi, P. Chitrakala, and A. Jenitha, "Face recognition based attendance marking system," International Journal of Computer Science and Mobile Computing, vol. 3, no. 2, pp. 337-342, February 2014.

[8] J. Freya, L. Pooja, P. Rhea, and M. Nikita, "Android based mobile attendance system," International Journal of Advance Research in Computer Science and Software Engineering, vol. 6, no. 2, pp. 369-371, February 2016.

[9] V. Pallavi and G. Namit, "Fingerprint based student attendance system using gsm," International Journal of Science and Research, vol. 2, no. 10, pp. 128-131, 2016.

[10] N. Sumita, P. Romin, and S. Tanvi, "RFID Technology Based Attendance Management System," International Journal of Computer, vol. 1, no. 1, pp. 516-521, 2013. 